\title{
The effect of flat and textured insoles on the balance of primary care elderly people: a randomized controlled clinical trial
}

This article was published in the following Dove Press journal:

Clinical Interventions in Aging

\author{
Cecília de Morais Barbosa' \\ Manoel Barros Bértolo² \\ Juliana Zonzini Gaino² \\ Michael Davitt ${ }^{3}$ \\ Zoraida Sachetto ${ }^{2}$ \\ Eduardo de Paiva \\ Magalhães ${ }^{3}$ \\ 'Department of Internal Medicine, \\ Gerontology, Faculty of Medical \\ Sciences, State University of \\ Campinas - Unicamp, Campinas, \\ SP, Brazil; ' 2 Department of Internal \\ Medicine, Rheumatology, Faculty of \\ Medical Sciences, State University of \\ Campinas - Unicamp, Campinas, SP, \\ Brazil; ${ }^{3}$ Orthoses and Prostheses Unit, \\ Clinical Hospital, State University of \\ Campinas - Unicamp, Campinas, SP, \\ Brazil
}

Background: Aging is associated with reduced postural stability and increased fall risk. Foot orthoses have been reported as an adjuvant intervention to improve balance by stimulating foot plantar mechanical receptors and thus increasing somatosensory input.

Purpose: The aim of this study is to evaluate the effect of flat and textured insoles on the balance of primary care elderly people.

Design: Prospective, parallel, randomized, and single-blind trial.

Methods: A total of 100 subjects from a primary care unit, aged $\geq 65$ years, were randomly assigned to intervention groups with flat insoles $(n=33)$, textured insoles $(n=33)$, or control group $(n=34)$ without insoles. The Berg Balance Scale and the Timed Up and Go test were assessed at baseline and after 4 weeks.

Results: Improvements in the Berg Balance Scale and the Timed Up and Go test were noted only in intervention groups with insoles but not in control group. No significant difference was found between flat and textured insoles. Minor adverse effects were noted only in the group with textured insoles.

Conclusion: The results suggest that foot orthoses (both flat and textured insoles) are effective in improving balance in primary care elderly people. They may represent a low-cost and highavailability adjuvant strategy to improve balance and prevent falls in this population.

Keywords: balance, elderly, orthoses, insole

\section{Introduction}

The incidence of falls and injuries increases with age. ${ }^{1,2}$ One-third of people aged $>65$ years fall every year and around $30 \%$ suffer moderate-to-severe injuries such as hip fractures, resulting in decline in function, hospitalization, medical costs, and increased mortality. ${ }^{1,3,4}$ Reducing fall risk in older individuals is, therefore, an important public health objective. ${ }^{5}$ Falls are generally related to abnormalities in gait and balance, which can be attributed, at least in part, to age-related impairments in the sensory system. ${ }^{6}$

The foot is the first point of contact between the body and the external environment, and it plays an important role in postural sway. ${ }^{7}$ Its plantar mechanical receptors provide spatial and temporal information about contact pressures and shear forces resulting from body movement being a valuable feedback mechanism to the postural control system. ${ }^{8}$ Interventions to optimize sensory information from plantar sole such as vibration stimuli, ${ }^{9-11}$ customized foot orthoses, ${ }^{12-15}$ textured insoles, ${ }^{16,17}$ and sandals with textured insoles ${ }^{18,19}$ and magnetic insoles ${ }^{20}$ have been tested before, some of them showing balance improvement. ${ }^{9-15}$

\section{Correspondence: Eduardo de Paiva Magalhães \\ Unidade de Órteses e Próteses, Hospital de Clínicas da Unicamp, Rua Vital Brazil 25I, Cidade Universitária Zeferino Vaz, Campinas, SP, I3083-888, Brazil \\ Email dreduardomagalhaes@gmail.com}


Textured insoles are simple interventions that could be a low-cost adjuvant intervention to improve balance in the elderly, without the need of podiatric assistance. There are just a few studies in this field, most of them with small number of patients and assessment of postural control soon after intervention. While balance improvement was observed with surface edge elevations, ${ }^{21,22}$ rounded plastic nubs, ${ }^{17}$ granulations, ${ }^{16}$ and sandals equipped with spike insoles,,${ }^{18,19}$ no difference was described with textured insoles in other studies. ${ }^{23-25}$

The aim of this study was to evaluate the effect of flat and textured insoles on the balance of primary care elderly people compared with a control group without orthoses.

\section{Methods}

\section{Design and study settings}

This was a randomized, controlled, single-blind clinical trial of the effect of 2 different insoles (flat or textured) on the balance of primary care elderly people compared with a control group without orthoses. It was conducted from March 2017 to June 2017 in a primary care unit in Campinas, São Paulo, Brazil. Trial registration: this trial has been registered in The Brazilian Clinical Trials Registry (ReBEC), http://ensaiosclinicos.gov. brensaiosclinicos.gov.br, RBR-8wfjw9.

\section{Ethical considerations}

This study was in accordance with the Declaration of Helsinki and was approved by the ethics committee of the Faculty of Medical Sciences of the State University of Campinas Unicamp (CAAE-41375314.0.0000.5404). All subjects provided a written informed consent before study.

\section{Subjects}

Eligible participants were adults aged $\geq 65$ years with the ability to read and write in Portuguese. Exclusion criteria were bedridden subjects, previous vestibular diseases, central nervous system pathologies (such as stroke, dementia, Parkinson, etc.), previous diagnosis of peripheral neuropathy, current use of benzodiazepines or tricyclic antidepressants, use of insoles in the last month, previous history of foot surgery, and amputation of the lower limbs. These data were obtained after revision of medical record or were selfreported. Subjects who had lower limb ulcers, committed tactile and thermal sensitivity, severe foot deformity (not able to wear regular shoes), difficulty in adapting insoles inside his/her own shoes, inability to attend the necessary reevaluations or follow instructions of the research protocol were also excluded. For tactile sensitivity, subjects were tested using a $10 \mathrm{~g}$ Semmes-Weinstein nylon monofilament (SORRI-Bauru, São Paulo, Brazil) as described previously. ${ }^{26}$ Subjects were instructed to say yes each time he/she perceived the application of the monofilament in 10 sites on the foot (plantar aspects of the first, third, and fifth digits; the plantar aspects of the first, third, and fifth metatarsal heads; the plantar medial and lateral sides of the midfoot; the plantar area of the heel; and the dorsal aspect of the midfoot). The inability to distinguish monofilament at 4 points or more was indicative of loss of protection sensation and resulted in exclusion of subject. For thermal sensitivity, specific lower limbs dermatomes (of the saphenous, superficial peroneal, deep peroneal, sural, posterior tibial, medial plantar, and lateral plantar nerves) were tested with 2 glass tubes: one filled with water, cooled and maintained at $7^{\circ} \mathrm{C}$, and the other heated and maintained at $40^{\circ} \mathrm{C} .{ }^{27}$ If subjects could not identify cold or heat in all regions, he/she was excluded.

\section{Intervention}

Subjects from the intervention groups received a flat or textured 3-mm thick insole made of ethylene-vinyl acetate (EVA; Technical Ltd, São Paulo, Brazil) with a flat (Shore value A 35 ) or textured surface (Shore Value A 40). The surface of the textured insole was entirely covered with small pyramidal peaks with center-to-center distances of $\sim 2 \mathrm{~mm}$ (Figure 1). The insoles were manufactured according to the subjects' shoe sizes in the Orthoses and Prostheses Unity of the Clinical Hospital of Campinas State University - Unicamp. Insoles were worn inside the subjects' own regular shoes.

\section{Outcome measures}

The primary outcome measures were the Berg Balance Scale (BBS) and the Timed Up and Go test (TUG) assessed at the initial visit and after 4 weeks.
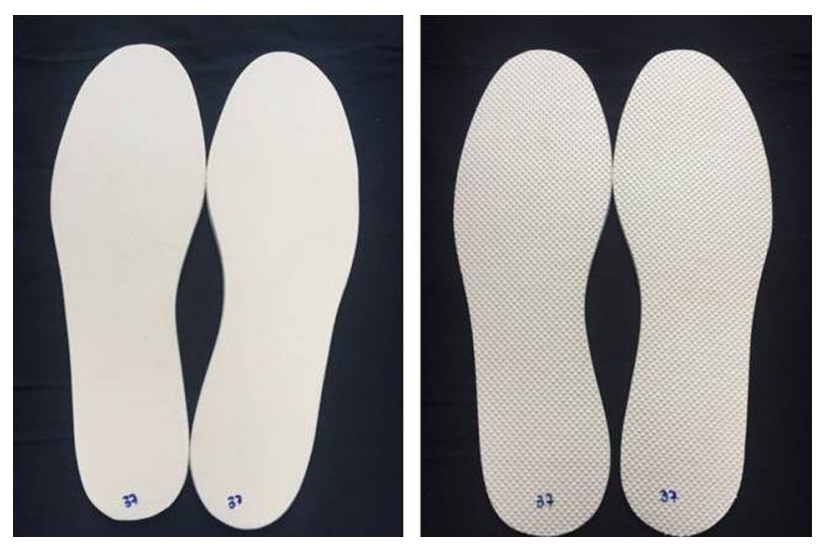

Figure I Flat insole (left) and textured insole (right). 
The BBS is a balance assessment test that rates the ability of a subject to maintain balance while performing 14 movements required in everyday activities (including transferring, standing unsupported, rising from a sitting to a standing position, tandem standing, turning $360^{\circ}$, and single-leg standing). Scoring is based on an ordinal 5-point scale of 0 to 4 . A score of 0 is given if the subject is unable to complete the task and a 4 is given if the subject is able to complete the task unassisted. The maximum possible score is 56 . Testing takes approximately $15-20$ minutes. $^{28}$

The TUG test is used to assess the dynamic balance of an individual. It measures the amount of time (recorded in seconds) it takes for individuals to rise from a standard armchair, walk a distance of 3 meters, and return to the initial position resting against the back of the chair. ${ }^{29}$

\section{Data collection}

Database from a primary care unit in Campinas/SP/Brazil was used for screening of the subjects. Medical records were analyzed regarding inclusion and exclusion criteria. Those considered eligible were invited to take part in the study through a telephone call or during their regular appointments. Those who agreed to participate had an appointment scheduled. Participants were asked to bring their own walking shoes. The study protocol was explained, and the subjects read and signed the informed consent. The subjects were checked again with regard to the eligible criteria. A sociodemographic and clinical questionnaire was applied concerning age, race, marital status, education, previous bone fracture, body mass index, use of drugs related to balance dysfunction (anticonvulsants, antidepressants, sedatives, and antihypertensives), number of falls in the last 12 months, and visual and hearing impairment. Lower limbs examination was performed considering the presence of ulcers, foot deformities, and foot tactile and thermal sensitivity.

All subjects answered the Mini Mental State Examination (MMSE), ${ }^{30}$ the Geriatric Depression Scale-Short Form (GDS), ${ }^{31}$ and the Manchester Foot Pain and Disability Index (MFPDI). ${ }^{32}$ Foot pain was assessed using a Visual Analog Pain Scale (VAS). ${ }^{33}$ Subjects were then randomized into intervention groups ( $\mathrm{G} 1$ - flat insoles, and G2 - textured insole) or control group ( $\mathrm{CG}$ - without insoles). Subjects from $\mathrm{G} 1$ and G2 received a pair of insoles - flat or textured, respectively. All of them were instructed to wear the insoles as often as they could, to record insole daily wearing time in a calendar and report discomfort or occurrence of any adverse effect related to this intervention. BBS and TUG were assessed at initial visit and after 4 weeks with own walking shoes of the subjects, without insoles. These tests were applied by a blinded assessor (a trained nurse) that was not aware of the subjects' allocation. Before being tested, each subject was asked to keep the group allocation in secret. The assessor restricted her speech to tests' instructions.

\section{Randomization}

Eligible subjects were randomly assigned to intervention groups with insoles (flat insoles - Group 1/G1 and textured insoles - Group 2/G2) or control group (CG) without orthoses. Randomization was performed using 100 sequentially numbered opaque envelopes with concealed group allocation. The envelopes were previously filled with the group description, which was printed on small papers and drawn from a closed bag.

\section{Sample size}

Sample size was calculated using analysis of variance (ANOVA) for repeated measurements with BBS and TUG values transformed into ranks. For this purpose, a pilot study was done with 61 subjects - 22 with textured insoles, 17 with flat insoles, and 22 without insoles. The number of the sample size for a power of $80 \%$ and a level of significance of $5 \%$ was 20 subjects in each group. Subjects from the pilot study were also included in the final evaluation.

\section{Statistics}

For sample profile analyses, tables with descriptive statistics showing the mean, SD, median, maximum, and minimum values of each variable were used.

The Kruskal-Wallis, chi-squared, and Fisher's exact tests were applied to compare baseline values between groups. Dunn's multiple comparison test was used to detect if there was a difference between groups. A comparison between groups and times was performed through ANOVA for repeated measures as variables transformed into ranks.

All the data were analyzed using Statistical Analysis System for Windows (version 9.4; SAS Institute Inc., Cary, $\mathrm{NC}, \mathrm{USA})$ with a $5 \%$ level of significance $(p<0.05)$.

\section{Results}

Figure 2 describes the study flowchart. The medical records of 838 subjects aged $\geq 65$ years currently registered in the primary care unit studied were analyzed taking into consideration the inclusion and exclusion criteria. A total of 549 subjects were excluded due to the inability to $\mathrm{read} /$ write $(n=295)$ or specific clinical conditions $(n=254)$. From the 


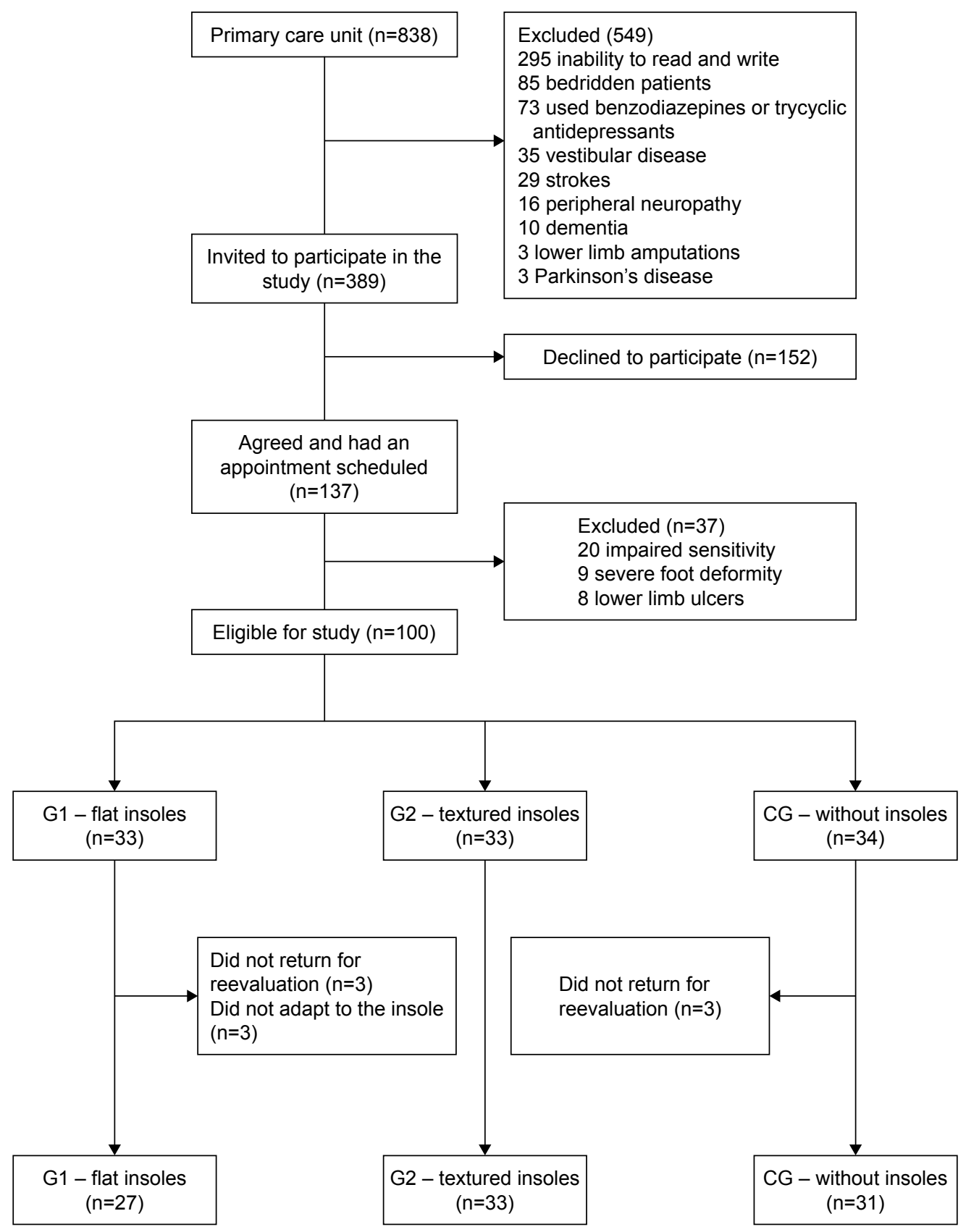

Figure 2 Study flowchart.

389 subjects invited to take part in the study, 152 declined, and 137 agreed and had an appointment scheduled. After clinical evaluation, 37 subjects were excluded as follows: 20 with impaired sensitivity, 9 could not wear insoles and shoes due to severe foot deformity, and 8 because of the presence of lower limb ulcers. One hundred subjects were then randomized, 33 in G1 - flat insoles, 33 in G2 - textured insoles, and 34 in $\mathrm{CG}$ - without insoles. Nine subjects did not complete the protocol, 6 from G1 (3 did not return due to personal reasons and 3 did not adapt to the intervention due to feeling hot in the feet when wearing the insole), and
3 from control group (did not return to reassessment due to personal reasons). Ninety-one subjects ( 27 G1, 33 G2, 31 CG) were used for final analysis.

Table 1 shows baseline characteristics of the subjects studied. No significant difference was found between groups at baseline considering the sociodemographic and clinical data, MMSE, GDS, MFPDI, VAS, BBS, and TUG.

Outcome measures (BBS and TUG) before and after intervention are shown in Table 2 and Figure 3. A significant improvement was noted after 4 weeks in both G1 and G2 $(p<0.0001)$, but not in CG ( $p=0.14$ and $p=0.64$ for BBS and 
Table I Baseline characteristics of subjects from GI - flat insoles, G2 - textured insoles, and CG - without insoles

\begin{tabular}{|c|c|c|c|c|c|}
\hline Characteristic & GI $(n=27)$ & G2 $(n=33)$ & CG $(n=3 I)$ & All participants $(n=9 I)$ & $p$-values \\
\hline Age (years)* & $69.4 \pm 4.1(69.0,65-82)$ & $70.1 \pm 4.5(70.0,65-84)$ & $70.4 \pm 5.4(68.0,65-86)$ & $70.0 \pm 4.7(69.0,65-86)$ & $0.83^{\mathrm{a}}$ \\
\hline \multicolumn{6}{|l|}{ Sex** } \\
\hline Male & $12(44.4)$ & 14 (42.4) & $16(51.6)$ & $42(46.2)$ & \multirow[t]{2}{*}{$0.75^{\mathrm{b}}$} \\
\hline Female & $15(55.6)$ & $19(57.6)$ & $15(55.6)$ & $49(53.8)$ & \\
\hline \multicolumn{6}{|l|}{ Race** } \\
\hline White & $15(55.6)$ & $16(48.5)$ & $20(64.5)$ & $20(64.5)$ & \multirow[t]{3}{*}{$0.67^{\mathrm{b}}$} \\
\hline Black & $8(529.6)$ & $19(57.6)$ & $7(22.6)$ & $7(22.6)$ & \\
\hline Mullato & $4(14.8)$ & $8(24.2)$ & $4(12.9)$ & $4(12.9)$ & \\
\hline \multicolumn{6}{|l|}{ Education (years) ${ }^{* *}$} \\
\hline$<4$ & $18(66.7)$ & I $8(54.5)$ & $19(61.3)$ & $55(60.4)$ & \multirow[t]{2}{*}{$0.63^{\mathrm{b}}$} \\
\hline $4-8$ & $9(33.3)$ & $15(45.5)$ & $12(38.7)$ & $36(39.6)$ & \\
\hline BMI $\left(\mathrm{kg} / \mathrm{m}^{2}\right)^{*}$ & $27.3 \pm 4.1(27.5,18.5-35.8)$ & $27.8 \pm 4.5(27.5,20.4-41.9)$ & $28.2 \pm 4.5(28.6,19.8-41.9)$ & $27.8 \pm 4.4(27.7,18.5-41.9)$ & $0.76^{a}$ \\
\hline $\begin{array}{l}\text { Drugs related to } \\
\text { balance** }\end{array}$ & $20(74.1)$ & $24(72.7)$ & $26(83.9)$ & $70(76.9)$ & $0.52^{\mathrm{b}}$ \\
\hline Visual impairment** & $24(88.9)$ & $25(75.8)$ & $22(71.0)$ & 71 (78.0) & $0.24^{b}$ \\
\hline Hearing impairment ${ }^{* *}$ & $5(18.5)$ & $9(27.3)$ & $4(12.9)$ & $18(19.8)$ & $0.35^{b}$ \\
\hline Occurrence of fractures $* *$ & $10(37.0)$ & $11(33.3)$ & $6(19.4)$ & 27 (76.9) & $0.29^{b}$ \\
\hline \multicolumn{6}{|c|}{ Falls in the past 12 months** } \\
\hline 0 & $19(70.4)$ & $19(57.6)$ & $21(67.7)$ & $59(64.8)$ & \multirow[t]{3}{*}{$0.65^{c}$} \\
\hline$<3$ & $8(29.6)$ & $12(36.4)$ & $8(25.8)$ & $28(30.8)$ & \\
\hline $3-5$ & $0(0.0)$ & $2(6.1)$ & $2(6.5)$ & $4(4.4)$ & \\
\hline MMSE* & $24.3 \pm 4.1(25.0,16-30)$ & $25.3 \pm 3.5(26.6,15-30)$ & $24.7 \pm 3.3(25.0,15-29)$ & $24.8 \pm 3.6(26.0,15-30)$ & $0.62^{\mathrm{a}}$ \\
\hline GDS* & $2.7 \pm 2.1(2.0,0-8)$ & $3.2 \pm 3.2(2.0,0-11)$ & $3.7 \pm 2.7(4.0,0-9)$ & $3.2 \pm 2.7(2.0,0-11)$ & $0.40^{\mathrm{a}}$ \\
\hline MFPDI* & $5.6 \pm 6.0(4.0,0-16)$ & $4.5 \pm 6.6(0.0,0-22)$ & $3.7 \pm 7.0(0.0,0-30)$ & $4.5 \pm 6.5(0.0,0-30)$ & $0.20^{\mathrm{a}}$ \\
\hline VAS* & $2.3 \pm 3.4(0.0,0-10)$ & $1.6 \pm 2.5(0.0,0-8.5)$ & $1.0 \pm 2.2(0.0,0-8)$ & $1.6 \pm 2.7(0.0,0-10)$ & $0.33^{\mathrm{a}}$ \\
\hline BBS* & $50.3 \pm 3.7(51.0,43-55)$ & $48.9 \pm 4.3(49.0,40-55)$ & $48.9 \pm 5.0(50.0,31-55)$ & $49.3 \pm 4.4(50.0,31-55)$ & $0.37^{\mathrm{a}}$ \\
\hline TUG* & $11.8 \pm 3.2(10.8,7.8-22.8)$ & $10.4 \pm 2.6(9.5,5.2-17.1)$ & $12.0 \pm 3.4(10.8,7.1-20.0)$ & $11.4 \pm 3.1(10.6,5.2-22.8)$ & $0.046^{\mathrm{a}, \#}$ \\
\hline
\end{tabular}

Notes: *Mean \pm SD (median, minimum value-maximum value). **Number (percentage). "Dunn's multiple-comparison test found no difference between group. ${ }^{*}$ Kruskal-

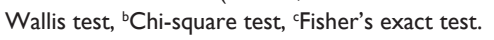

Abbreviations: BBS, Berg Balance Scale; BMI, body mass index; CG, control group; GDS, Geriatric Depression Scale; MFPDI, Manchester Foot Pain and Disability Index; MMSE, Mini Mental State Examination; TUG, Timed UP and Go test; VAS, Visual Analog Pain Scale.

TUG, respectively) (Figure 3). No difference could be noted between G1 and G2.

Subjects referred they wore the insoles during a mean of 26.6 days $( \pm 1.6,24-28)$ in $\mathrm{G} 1$ and 25.9 days $( \pm 3.4,12-28)$

Table 2 Values of BBS and TUG at baseline $\left(\mathrm{t}_{0}\right)$ and after 4 weeks $\left(t_{1}\right)$ from $\mathrm{GI}$ - flat insoles, G2 - textured insoles, and CG - without insoles

\begin{tabular}{llll}
\hline Variables & $\mathbf{t}_{\mathbf{0}}$ & $\mathbf{t}_{\mathbf{l}}$ & $\mathbf{p}$-value* \\
\hline $\mathrm{GI}_{\text {BBS }}$ & $50.3 \pm 3.7$ & $53.5 \pm 2.3 \mathrm{I}$ & $<0.000 \mathrm{I}$ \\
& $(51.0,43-55)$ & $(54.0,48-56)$ & \\
$\mathrm{GI}_{\text {TUG }}$ & $1 \mathrm{I} .8 \pm 3.2$ & $9.5 \pm 2.1$ & \\
& $(10.8,7.8-22.8)$ & $(9.3,6.6-14.6)$ & \\
$\mathrm{G}_{\text {BBS }}$ & $48.9 \pm 4.3$ & $53.4 \pm 2.9$ & $<0.000 \mathrm{I}$ \\
$\mathrm{G2}_{\text {TUG }}$ & $(49.0,40-55)$ & $(54.0,46-56)$ & \\
$\mathrm{CG}_{\text {BBS }}$ & $10.4 \pm 2.6$ & $8.5 \pm 1.8$ & \\
$\mathrm{CG}_{\text {TUG }}$ & $(9.5,5.2-17.1)$ & $(8.3,5.8-14.4)$ & \\
& $48.9 \pm 5.0$ & $48.5 \pm 5.0$ & 0.1466 \\
\hline
\end{tabular}

Notes: Data shown as mean \pm SD (median, minimum value-maximum value). *Analysis of variance for repeated measures.

Abbreviations: BBS, Berg Balance Scale; CG, control group; TUG, Timed Up and Go test. in G2. Insole wearing time was $6 \mathrm{~h} /$ day $( \pm 2.4,2.2-12)$ in $\mathrm{G} 1$ and $6.2 \mathrm{~h} /$ day $( \pm 2.7,1.9-12)$ in G2. No significant difference was found between groups $(p=0.91)$. The number of days using orthoses and insoles wearing time did not interfere in outcome measures (BBS and TUG).

As shown in the flowchart (Figure 2), minor adverse effect was noted only in G1 where 3 subjects did not use the insole due to feeling hot in the feet.

\section{Discussion}

In this study, we aimed to investigate the effect of insoles with a flat or textured surface on the balance of primary care elderly people. After 4 weeks, outcome measures (BBS and TUG) improved in intervention groups (with flat or textured insoles), but not in control group without orthoses.

Foot orthoses have been reported as an interesting adjuvant intervention to improve postural sway and prevent falls by stimulating foot plantar mechanical receptors and thus increasing somatosensory input. ${ }^{9-22}$ Interventions include vibration insoles, ${ }^{9-11}$ customized foot orthoses, ${ }^{12-15}$ and insoles with a textured surface. ${ }^{16,17}$ Concerning insoles with vibration 

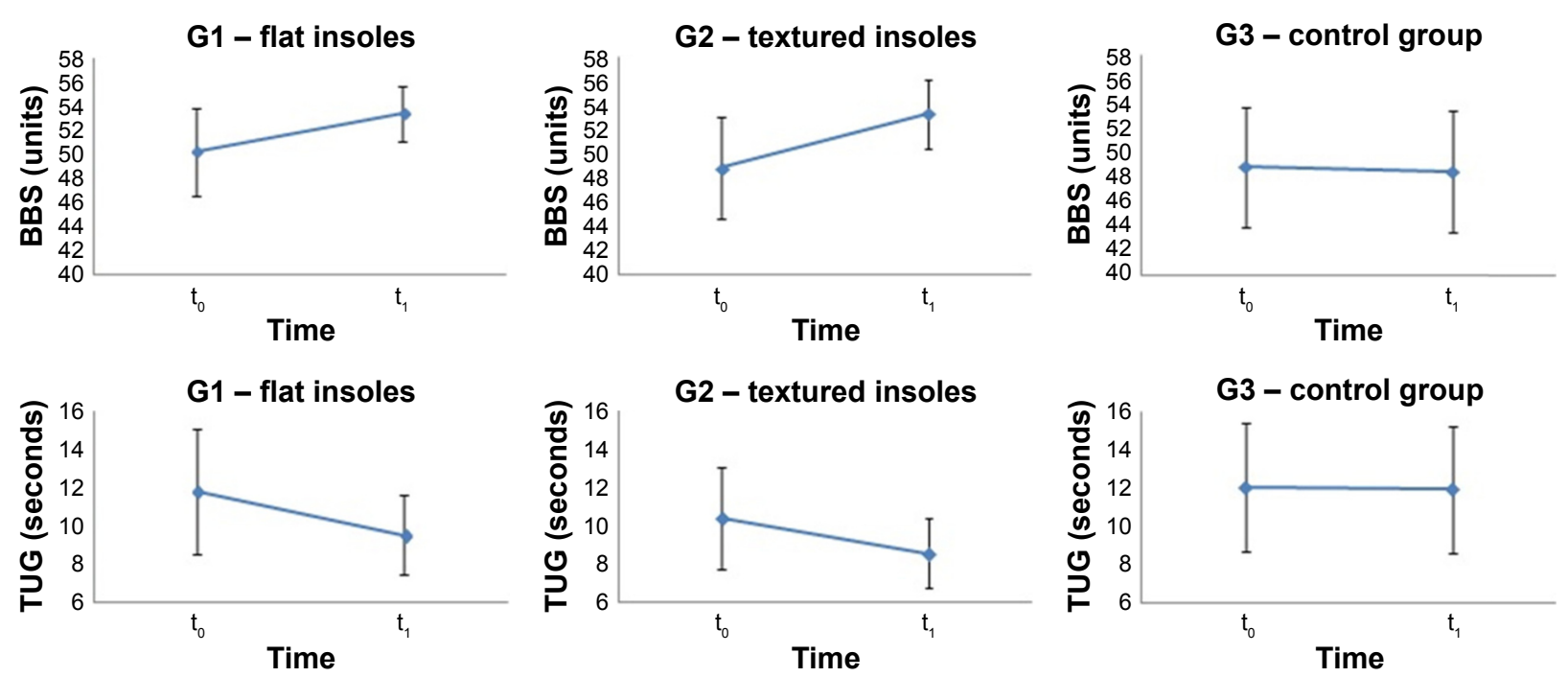

Figure 3 BBS and TUG in groups with flat (GI) or textured insoles (G2) in comparison with the control group (G3) without orthoses from the beginning ( $\left.\mathrm{t}_{0}\right)$ to the end of the 4 weeks of the protocol $\left(t_{1}\right)$.

Abbreviations: BBS, Berg Balance Scale; TUG, Timed Up and Go test.

stimuli, studies are promising with some evidence of balance improvement in elderly people ${ }^{9-11}$ and subjects with pathologies such as stroke, diabetes, ${ }^{34}$ and ankle instability. ${ }^{35}$ The number of subjects in these studies were small, and the balance tests were applied soon after intervention. Therefore, it is uncertain if this immediate amelioration would be sustained. The cost and feasibility of these vibratory devices may also be a limitation for its widespread use. ${ }^{13}$

Customized foot orthoses have also been investigated with some evidence of balance improvement after short-time follow-up (2-8 weeks). ${ }^{12-15}$ Interventions included individual prescriptions,${ }^{13}$ insoles with an arch support, ${ }^{15}$ and a heel cup with arch support. ${ }^{12}$ Previously, we have also observed a balance improvement (BBS and TUG) in a 4-week randomized controlled trial with 89 elderly woman with osteoporosis using an insole with arch support and a metatarsal pad. ${ }^{14}$ Although these orthoses are commonly available and well accepted, podiatric support may be necessary.

Surface insole modifications could be a simpler intervention compared with vibration or customized insoles. There are some studies of balance using textures over the insole or shoes with different results. In one of them, Maki et al observed balance improvement in both young and older subjects using an insole with edge elevations. ${ }^{21}$ In another, Corbin et al observed better postural control in adults when wearing textured insoles, with small rounded plastic nubs. ${ }^{17}$ Improvements in postural sway of elderly subjects were also described by Qiu et al using hard or soft insole with small granulations over its surface, ${ }^{16}$ and in 2 similar studies of Palluel et al using sandals equipped with spike insoles. ${ }^{18,19}$ On the contrary, Hatton et al observed no difference in static balance in older fallers using a textured or smooth insole although gait velocity was reduced with textured insoles; step and stride lengths were smaller with textured insoles when compared with smooth insoles. ${ }^{23}$ Also, Qu when studying different foot orthoses - cupped, textured, rigid, and soft insoles, did not observe any difference in static stability, and only the cupped insole improved dynamic postural stability. ${ }^{24}$ All of these studies had a small number of subjects, and balance analysis was performed soon after intervention.

There are 2 studies with textured insoles with a short period of follow-up. In 1 of them, Perry et al described improvement in lateral stability during gait and reduction in falls in 40 community-dwelling older adults after a 12-week-protocol using a "facilitatory" insole (with edge elevation). ${ }^{22}$ In other, Wilson et al did not find significant difference in postural stability when studying 40 healthy women (aged 51.1 \pm 5.8 years) with 4 different intervention groups (plain orthoses, dimple orthoses, grid orthoses, and control group) after a period of 4 weeks. ${ }^{25}$ In our study, a flat EVA - commonly used as the base of customized foot orthoses - and a textured EVA - mainly used as slippers soles - were used. Subjects were evaluated after 4 weeks and benefits were evident.

No data are available regarding the frequency of insole use necessary to achieve balance sway improvement. As shown earlier, evidences of postural sway improvement were observed soon after intervention. ${ }^{16,17,21}$ Here, insole wearing time did not influence BBS and TUG response. Probably, benefits could be seen even if wearing foot orthoses for brief 
periods. Even though the insole wearing time necessary to achieve the best results is still to be determined.

In addition, it is still uncertain if results would be sustained during long periods of follow-up. ${ }^{36}$ In this study, benefits were observed after 4 weeks. There are some other evidences of sustained balance improvement after periods varying from 2 to 12 weeks. ${ }^{12-16}$

Many scales are used for balance assessment. In this study, we used BBS and TUG, which are known as good instruments to estimate the risk of fall in the elderly with high intra- and inter-rater reliability. They are also easy to apply and interpret. ${ }^{37,38}$ Although many authors use posturography and gait analysis for monitoring the effect of interventions on balance, these methods have some important limitations related to cost, space, and the need of a trained professional. So, they were not used in this protocol.

This study has some limitations. It would be reasonable to expect that only the texture insole would provide skin mechanical stimulations and that the flat insole would work as a placebo or disclose a minor effect. On the contrary, balance improvement was similar in both intervention groups. Adverse effects (feeling hot in the feet) were also noted only in the flat insole group. Thus, both insoles may have improved sensory output favoring a better postural control. Since it is difficult to consider a suitable placebo insole, it has been suggested to use a control group without any intervention, ${ }^{39,40}$ as we did. Unfortunately, lack of an intervention in the control group might have generated a resentful demoralization (when participants allocated to a no-treatment control group may be resentful of not receiving an intervention) and ascertainment bias (when results/conclusions are distorted by the knowledge of which intervention the participant is receiving) ${ }^{41}$ So, a placebo effect in the intervention groups cannot be ruled out.

A second limitation is that footwear was not controlled in this study. The subjects were asked to use their own shoes. The use of shoes suitable for the use of insoles could also have favored improvements in intervention groups ${ }^{42,43}$ Use of the same shoes during a long period of time may hamper the enrollment of the subjects and increase dropouts.

Finally, we cannot assure that the balance improvement observed in this study would translate into a reduced number of falls. Also, it is uncertain if subjects would continue wearing insoles and benefits would be sustained for longer periods of time.

Despite these considerations, we believe that the improvements observed in the balance in this study, as in others from literature, may not be neglected. Insoles are generally well accepted with no important adverse effect. They may represent a feasible adjuvant intervention to improve balance, thus reducing the risk of falls and its adverse outcomes in morbidity and mortality in elderly.

\section{Conclusion}

In this randomized controlled clinical trial, we observed improvements in the balance of subjects wearing flat or textured insoles. These interventions may represent a low-cost and high-availability adjuvant method to improve balance in community-dwelling elderly people. Prospective studies in this field should be encouraged to confirm its benefits over long periods, especially with regard to fall prevention.

\section{Acknowledgments}

We would like to thank Edvandro Lima Batista and Antonio Marcos Freitas Aquino from the Ortheses and Prostheses Unit; Juliana Luz Passos Argenton and Cleide Aparecida Moreira da Silva for the statistical analysis; and Ana Lúcia de Morais Barbosa for her help in data acquisition.

\section{Author contributions}

All authors contributed toward data analysis, drafting and critically revising the paper, gave final approval of the version to be published, and agree to be accountable for all aspects of the work.

\section{Disclosure}

The authors report no conflicts of interest in this work.

\section{References}

1. Centers for Disease Control and Prevention. Self-reported falls and fall-related injuries among persons aged $\geq 65$ years - United States, 2006. MMWR Morb Mortal Wkly Rep. 2008;57(9):225-229.

2. Rubenstein LZ, Josephson KR. The epidemiology of falls and syncope. Clin Geriatr Med. 2002;18(2):141-158.

3. National Center for Injury Prevention and Control. Ten Leading Causes of Death By Age Group, United States - 2003. Atlanta, GA: Centers for Disease Control and Prevention; 2007.

4. Fuller GF. Falls in the elderly. Am Fam Physician. 2000;61(7): 2159-2168.

5. Sattin RW. Falls among older persons: a public health perspective. Annu Rev Public Health. 1992;13:489-508.

6. Sturnieks DL, St George R, Lord SR. Balance disorders in the elderly Neurophysiol Clin. 2008;38(6):467-478.

7. Maurer C, Mergner T, Bolha B, Hlavacka F. Human balance control during cutaneous stimulation of the plantar soles. Neurosci Lett. 2001;302(1):45-48.

8. Kavounoudias A, Roll R, Roll JP. The plantar sole is a 'dynamometric map' for human balance control. Neuroreport. 1998;9(14):3247-3252.

9. Priplata AA, Niemi JB, Harry JD, Lipsitz LA, Collins JJ. Vibrating insoles and balance control in elderly people. Lancet. 2003;362(9390): $1123-1124$.

10. Galica AM, Kang HG, Priplata AA, et al. Subsensory vibrations to the feet reduce gait variability in elderly fallers. Gait Posture. 2009; 30(3):383-387. 
11. Lipsitz L, Lough M, Niemi J, Travison T, Howlett H, Manor B. A shoe insole delivering subsensory vibratory noise improves balance and gait in healthy elderly people. Arch Phys Med Rehabil. 2015;96(3): 432-439.

12. Chen TH, Chou LW, Tsai MW, Lo MJ, Kao MJ. Effectiveness of a heel cup with an arch support insole on the standing balance of the elderly. Clin Interv Aging. 2014;9:351-356.

13. Gross MT, Mercer VS, Lin FC. Effects of foot orthoses on balance in older adults. J Orthop Sports Phys Ther. 2012;42(7):649-657.

14. de Morais Barbosa C, Barros Bértolo M, Marques Neto JF, Bellini Coimbra I, Davitt M, de Paiva Magalhães E. The effect of foot orthoses on balance, foot pain and disability in elderly women with osteoporosis: a randomized clinical trial. Rheumatology (Oxford). 2013;52(3): $515-522$.

15. Mulford D, Taggart HM, Nivens A, Payrie C. Arch support use for improving balance and reducing pain in older adults. Appl Nurs Rev. 2008;21(3):153-158.

16. Qiu F, Cole MH, Davids KW, et al. Enhanced somatosensory information decreases postural sway in older people. Gait Posture. 2012; 35(4):630-635.

17. Corbin DM, Hart JM, McKeon PO, Ingersoll CD, Hertel J. The effect of textured insoles on postural control in double and single limb stance. J Sport Rehabil. 2007;16(4):363-372.

18. Palluel E, Olivier I, Nougier V. Do spike insoles enhance postural stability and plantar-surface cutaneous sensitivity in the elderly? Age (Dordr). 2008;30(1):53-61.

19. Palluel E, Olivier I, Nougier V. The lasting effects of spike insoles on postural control in the elderly. Behav Neurosci. 2009;123(5):1141-1147.

20. Suomi R, Koceja DM. Effect of magnetic insoles on postural sway measures in men and women during a static balance test. Percept Mot Skills. 2001;92(2):469-476.

21. Maki BE, Perry SD, Norrie RG, Mcllroy WE. Effect of facilitation of sensation from plantar foot-surface boundaries on postural stabilization in young and older adults. J Gerontol A Biol Sci Med Sci. 1999; 54(6):M281-M287.

22. Perry SD, Radtke A, Mcilroy WE, Fernie GR, Maki BE. Efficacy and effectiveness of a balance-enhancing insole. J Gerontol A Biol Sci Med Sci. 2008;63(6):595-602.

23. Hatton AL, Dixon J, Rome K, Newton JL, Martin DJ. Altering gait by way of stimulation of the plantar surface of the foot: the immediate effect of wearing textured insoles in older fallers. J Foot Ankle Res. 2012; 30:5-11.

24. Qu X. Impacts of different types of insoles on postural stability in older adults. Appl Ergon. 2015;46(Pt A):38-43.

25. Wilson ML, Rome K, Hodgson D, Ball P. Effect of textured foot orthotics on static and dynamic postural stability in middle-aged females. Gait Posture. 2008;27(1):36-42.

26. Mueller MJ. Identifying patients with diabetes mellitus who are at risk for lower-extremity complications: use of Semmes-Weinstein monofilaments. Phys Ther. 1996;76(1):68-71.
27. Batista F, Pinzur MS, Nery CA. Cutaneous thermal sensitivity in diabetic neuropathy. Foot Ankle Int. 2005;26(11):927-931.

28. Berg K, Wood-Dauphinee S, Williams J. The Balance Scale: reliability assessment with elderly residents and patients with an acute stroke. Scand J Rehabil Med. 1995;27(1):27-36.

29. Podsiadlo D, Richardson S. The timed "Up \& Go": a test of basic functional mobility for frail elderly persons. J Am Geriatr Soc. 1991; 39(2):142-148

30. Folstein MF, Folstein SE, McHugh PR. Mini-mental state: a practical method for grading the cognitive state of patients for the clinic. J Psychiatr Res. 1975;12(3):189-198.

31. Sheikh JI, Yesavage JA. Geriatric depression scale (GDS): recent evidence and development of a shorter version. Clin Gerontol. 1986; 33(3):219-228.

32. Menz HB, Tiedemann A, Kwan MM, Plumb K, Lord SR. Foot pain in community-dwelling older people: an evaluation of the Manchester Foot Pain and Disability Index. Rheumatology. 2006;45(7):863-867.

33. Huskisson EC. Visual Analogue Scales. In Melzack, R. Pain Measurement and Assessment. New York, NY: Raven Press; 1983:33-37.

34. Liu W, Lipsitz LA, Montero-Odasso M, Bean J, Kerrigan DC, Collins JJ. Noise-enhanced vibrotactile sensitivity in older adults, patients with stroke, and patients with diabetic neuropathy. Arch Phys Med Rehabil. 2002;83(2):171-176.

35. Ross SE. Noise-enhanced postural stability in subjects with functional ankle instability. Br J Sports Med. 2007;41(10):656-659.

36. Dixon J, Hatton AL, Robinson J, et al. Effect of textured insoles on balance and gait in people with multiple sclerosis: an exploratory trial. Physiotherapy. 2014;100(2):142-149.

37. Yelnik A, Bonan I. Clinical tools for assessing balance disorders. Neurophysiol Clin. 2008;38(6):439-445.

38. Hayes KW, Johnson ME. Measures of adult general performance tests The Berg Balance Scale, Dynamic Gait Index (DGI), Gait Velocity, Physical Performance Test (PPT), Timed Chair Stand Test, Timed Up and Go, and Tinetti Performance-Oriented Mobility Assessment (POMA). Arthritis Care Res. 2003;49(S5):S28-S42.

39. Conrad KJ, Budiman-Mak E, Roach KE, et al. Impacts of foot orthoses on pain and disability in rheumatoid arthritics. J Clin Epidemiol. 1996;49(1):1-7.

40. Lewinson RT, Stefanyshyn DJ. Losing control over control conditions in knee osteoarthritis orthotic research. Contemp Clin Trials. 2015; 42:258-259.

41. Bonanno DR, Landorf KB, Murley GS, Menz HB. Selecting control interventions for use in orthotic trials: the methodological benefits of sham orthoses. Contemp Clin Trials. 2015;42:257.

42. Lewinson RT, Worobets JT, Stefanyshyn DJ. Control conditions for footwear insole and orthotic research. Gait Posture. 2016;48:99-105.

43. Arnadottir SA, Mercer VS. Effects of footwear on measurements of balance and gait in women between the ages of 65 and 93 years. Phys Ther. 2000;80(1):17-27.
Clinical Interventions in Aging

\section{Publish your work in this journal}

Clinical Interventions in Aging is an international, peer-reviewed journal focusing on evidence-based reports on the value or lack thereof of treatments intended to prevent or delay the onset of maladaptive correlates of aging in human beings. This journal is indexed on PubMed Central, MedLine,
Dovepress

CAS, Scopus and the Elsevier Bibliographic databases. The manuscript management system is completely online and includes a very quick and fair peer-review system, which is all easy to use. Visit http://www.dovepress. com/testimonials.php to read real quotes from published authors. 